Reprod. Nutr. Dévelop., 1980, 20 (4 B), 1325-1329.

\title{
Actidione-cycloheximide ef voie d'absorption intestinale des lipides chez le rat
}

\author{
par Marinette BONDOUX-REBOUILLAT, A. BERNARD, Hélène CARLIER \\ avec la collaboration technique de Marie-France GIRARDIER et Marie-Claude MONNOT \\ Laboratoire de Physiologie de la Nutrition LA no 273 \\ ENS. BANA, Campus Montmuzard 21100 Dijon, France. \\ et UER Nutrition.
}

Summary. Actidione-cycloheximide and the intestinal lipid absorption route in the rat.

The effect of actidione-cycloheximide on the intestinal absorption of ${ }^{14} \mathrm{C}$ oleic acid was studied on male rats using the ex vivo perfusion technique in control rats and those treated with a protein synthesis inhibitor.

With the amounts of lipid infused, the radioactivity recovered in portal blood lipids was the same in treated rats and in controls.

However, differences were observed in the appearance of oleic acid in the portal blood during the first $15 \mathrm{~min}$. blood.

When protein synthesis was inhibited, absorption occurred earlier via the portal

The absorption balance in this type of experiment was considerably lower than normal absorption probably because or surgical stress.

In conclusion, when esterification was impaired, oleic acid seemed to be absorbed via the portal blood at the beginning of the experiment like short or medium-chain fatty acids.

\section{Introduction.}

On admet que la voie lymphatique est la voie normale d'absorption des acides gras à longue chaîne (Bloom, Chaikoff and Reinhardt, 1951 ; Bloomstrand, 1955). Toutefois la voie portale pourrait devenir la voie prépondérante lorsqu'il y a défauł d'estérification dans la muqueuse, comme le suggèrent Hyun, Vahouny et Treadwell (1967). Or, certains inhibiteurs de la synthèse protéique tels que l'actidione-cycloheximide diminuent nettement l'estérification de ces acides gras dans l'entérocyte (résultats non publiés) ; une faible fraction seulement des lipides absorbés étant retrouvée dans la lymphe (Bernard et al., 1979), la voie lymphatique ne représente donc plus la voie majeure d'absorption des acides gras à longue chaîne. Le défaut d'estérification dans la muqueuse du rat traité, entraîne-t-il alors une absorption de ces acides gras par la voie sanguine? 
Ce travail représente une première approche qui devrait contribuer à répondre à la question posée. Pour cela on utilise une technique de perfusion « ex vivo » permettant de prélever continuellement le sang d'origine mésentérique au cours de l'absorption des lipides.

\section{Matériel et méthodes.}

Animaux. - On utilise des rats mâles adultes Wistar pesant 180 à $200 \mathrm{~g}$. Les animaux sont mis à jeun la veille de l'expérience, gardant libre accès à l'eau de boisson.

Les rats traités reçoivent, $4 \mathrm{~h}$ avant l'expérience, une injection intrapéritonéale de $1,6 \mathrm{mg} / \mathrm{kg}$ d'actidione-cycloheximide (Boehringer).

Protocole expérimental. - Sous légère anesthésie à l'éther, on pratique une laparotomie et on met en place la perfusion « ex vivo» adaptée d'Eloy ef al. (1977). Cette technique permet de collecter continuellement la totalité du sang d'origine mésentérique. Afin de compenser la perte importante de sang récupéré par la canule posée dans la veine porte, il est nécessaire de perfuser du sang isologue. La mise en place de la perfusion se fait par la succession des opérations suivantes :

- pose d'un cathéter (Biotrol no 4 ) dans l'aorte abdominale infrarénale. Ce cathéter est relié à un capteur de pression (Statham P 23 Db) couplé à un enregistreur Racia ; - pose dans une veine jugulaire d'un cathéter relié à une pompe péristaltique (minipuls Gilson) qui permettra la perfusion de sang isologue (le sang perfusé est obtenu par ponction cardiaque de rats donneurs de même souche mis à jeun $24 \mathrm{~h}$ ) ; — isolement « in situ » d'une anse intestinale de 15 à $20 \mathrm{~cm}$, entre 2 canules métalliques Collin-Gentille $n^{\circ} 18$. La canule supérieure est placée juste après l'abouchement du canal pancréatobiliaire dans le duodénum ;

- pose d'une canule métallique Collin-Gentille $n^{0} 9$ dans la veine-porte en vue de la collecte du sang.

Dès la canulation de la veine-porte, on perfuse du sang à $37^{\circ} \mathrm{C}$ à un débit tel que la pression artérielle soit équilibrée à la valeur initiale. Lorsque la pression est stabilisée à $90 \mathrm{~mm}$ de mercure, on introduit le milieu lipidique dans l'anse intestinale isolée et on collecte le sang par intervalles de $5 \mathrm{~min}$ pendant $60 \mathrm{~min}$.

Milieu lipidique. - Il est constitué d'un mélange équimoléculaire de $30 \mu \mathrm{mol}$ d'acide palmitique, $30 \mu \mathrm{mol}$ d'acide oléique, $30 \mu \mathrm{mol}$ d' $\alpha$-monopalmitine et de $10 \mu \mathrm{Ci}$ d'acide oléique ${ }^{14} \mathrm{C}$ marqué sur le carboxyle (CEA Saclay, France).

Le mélange est additionné de $1,5 \mathrm{ml}$ de bile d'un rat donneur. L'ensemble est émulsionné en présence d'éther éthylique, évaporé au bain-marie à $37^{\circ} \mathrm{C}$ de manière à obtenir une émulsion stable.

Extraction des lipides. - Les lipides sont extraits du reste de l'émulsion lipidique infusée, du contenu intestinal et des liquides de rinçage, de la muqueuse intestinale ef du sang par le diméthoxy méthane-méthanol 4/1 V/V (Delsal, 1944), déshydratés à l'alcool absolu ef repris par du chloroforme.

Les mesures de radioactivité sont effectuées à l'aide d'un appareil à scintillation liquide Packard Prias PLD Tri-Carb. 


\section{Résulfats.}

1. Les bilans d'absorption sont représentés dans le tableau 1.

Malgré les écarts constatés, il semble que le prélèvement par la muqueuse ainsi que la disparition des lipides de la muqueuse sont moindres chez les rats traités. En revanche, la radioactivité retrouvée au niveau des lipides du sang collecté est du même ordre de grandeur pour les deux lots d'animaux.

TABLEAU 1

\begin{tabular}{ccccc}
\hline & \multicolumn{3}{c}{ Pourcentage de radioactivité } \\
\cline { 2 - 5 } & $\begin{array}{c}\text { prélevée } \\
\text { par la muqueuse } \\
\text { dans la lumière } \\
\text { intestinale }\end{array}$ & $\begin{array}{c}\text { présente } \\
\text { dans la } \\
\text { muqueuse }\end{array}$ & $\begin{array}{c}\text { disparue } \\
\text { de la } \\
\text { muqueuse }\end{array}$ & $\begin{array}{c}\text { retrouvée } \\
\text { dans le sang }\end{array}$ \\
\hline $\begin{array}{c}\text { Rats témoins } \\
n=3\end{array}$ & $55,5 \pm 4,9$ & $27,7 \pm 5,7$ & $27,8 \pm 9,9$ & $2,2 \pm 0,5$ \\
\hline $\begin{array}{c}\text { Rats traités } \\
n=3\end{array}$ & $48,4 \pm 5,6$ & $25,8 \pm 3,4$ & $22,6 \pm 3,5$ & $1,9 \pm 0,5$ \\
\hline
\end{tabular}

Les valeurs (moyennes \pm écarts types) sont exprimées en pourcentage de radioactivité par rapport à la radioactivité introduite $; n=$ nombre d'animaux.

2. Répartition de la radioactivité dans le sang portal au cours de 60 min après infusion du milieu lipidique (fig. 1). - Les résultats sont exprimés par périodes de 5 min en pourcentages de radioactivité par rapport à la radioactivité totale refrouvée dans le sang.
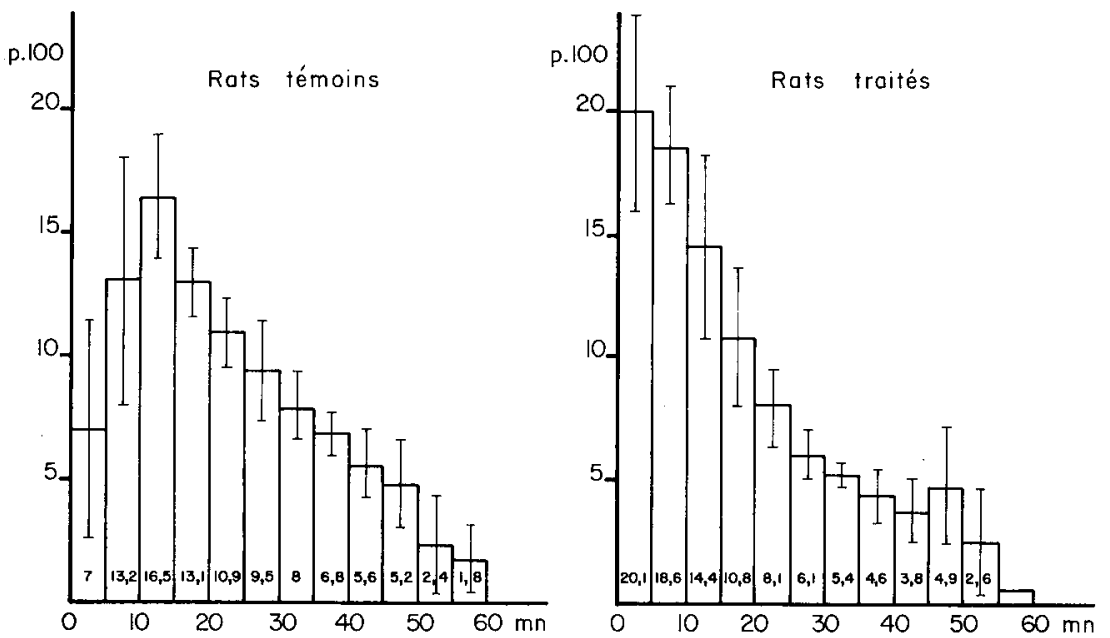

FIG. 1. - Pourcentage de radioactivité dans le sang de la veine-porte callecté par période de $5 \mathrm{~min}$, par rapport à la radioactivité totale retrouvée dons le sang. 
On remarque une évolution différente de la quantité de radioactivité entre les deux lots principalement dans les 15 premières minutes. Le pourcentage de radioactivité véhiculée par le sang atteint 20 et 18,5 p. 100 chez les animaux traités au cours des 2 premières collectes au lieu de seulement 7 p. 100 et 13,2 p. 100 chez les fémoins. En $15 \mathrm{~min}, 53$ p. 100 de la radioactivité sont déjà absorbés contre 36,9 p. 100 chez les témoins.

A partir de la 4 e période, l'évolution est sensiblement la même pour les deux lots d'animaux avec cependant un niveau légèrement plus élevé chez les témoins.

\section{Discussion.}

L'observation des résultats conduit à deux remarques concernant d'une part, le bilan d'absorption et d'autre part la voie d'absorption.

- Le pourcentage de radioactivité disparue du contenu intestinal et de la muqueuse est en $60 \mathrm{~min}$ nettement inférieur à ce qu'observaient Carlier ef Bézard (1975) et Bernard (non publié) (27,8 p. 100 au lieu de 74 p. 100 et 78 p. 100 respectivement dans des conditions voisines et en $30 \mathrm{~min}$ seulement). Cette faible absorption est peut-être due au choc opératoire et en particulier à une irrigation déficiente au niveau intestinal : on constate en effeł qu'il esł très difficile de maintenir la pression artérielle à son niveau normal touf au long de l'expérience, même en augmentant le débit de perfusion lorsque la pression artérielle diminue. Cette pression qui est de l'ordre de $90 \mathrm{~mm}$ de $\mathrm{Hg}$ au temps 0 tend à diminuer assez rapidement pour se stabiliser autour de $50 \mathrm{~mm}$ de $\mathrm{Hg}$.

- La radioactivité totale retrouvée dans le sang ne représente que 8 à 9 p. 100 de la radioactivité absorbée. Si ce comportement des animaux témoins vis-à-vis de l'acide gras à longue chaîne ne semble pas troublant, il pose un problème dans le cas des animaux traités à l'actidione-cycloheximide. Selon Hyun, Vahouny et Treadwell (1967), il semble que dans le cas d'une estérification déficiente, les acides gras à longue chaine puissent être absorbés par la voie sanguine. Si dans notre cas nous observons bien une diminution de l'absorption après l'inhibition de la synthèse protéique, cela ne semble pas entraîner une dérivation des lipides absorbés vers la voie sanguine. Ceci pourraît être dû au faił que le milieu lipidique infusé ne contient que $27 \mathrm{mg}$ de lipides. Ceci est à rapprocher des résultats de Glickman, Kirsch ef Isselbacher (1972) qui avec $42 \mathrm{mg}$ de lipides observent, dans la lymphe, après inhibition de la synthèse protéique, encore 42 p. 100 de la radioactivité absorbée et une augmentation de la taille des chylomicrons.

Par ailleurs, Redgrave (1969) constate que l'actidione-cycloheximide entraîne une diminution du flux lymphatique. Bernard et al. (1979) constatent une très forte diminution de la quantité de lipides retrouvés au niveau de la lymphe lors de l'absorption d'un milieu nettement plus chargé en lipides (0,05 p. 100 au lieu de 3 p. 100 chez les témoins). Il se pourrait donc que le stock enzymatique et en apoprotéine soit suffisant pour former encore des chylomicrons lorsque la quantité de lipides infusée est faible.

- L'évolution de la répartition de la radioactivité véhiculée par le sang portal présente une nette différence entre les deux lots d'animaux. Le passage des lipides 
dans le sang est plus précoce et plus important chez les animaux traités (20 p. 100 dans les 5 premières minutes au lieu de 7 p. 100 chez les témoins et 18 p. 100 entre la 5 e et la 10 e minute au lieu de 13 p. 100). Cette évolution est à rapprocher des résultats de Hyun, Vahouny et Treadwell (1967) et de ceux de Carlier et Bézard (1975) obtenus lors d'absorption d'acides gras à chaîne courte ou moyenne.

Ces premiers résultats semblent indiquer que l'acide oléique se comporte dans les conditions d'inhibition de la synthèse protéique, comme un acide gras à chaîne moyenne en empruntant plus rapidement la voie portale que dans les conditions normales, bien que la quantité de lipides infusée soił faible. Afin de confirmer ceci, il nous paraît indispensable, cependant, de mieux contrôler les conditions opératoires.

\author{
Journées Ingestion-Digestion-Absorption \\ de l'Association française de Nutrition, \\ Paris, 15-16 novembre 1979.
}

\title{
Références
}

BERNARD A., CARLIER H., CASELLI C., BÉZARD J., 1979. Effect of acetocycloheximide (A-CH) on infestinal absorption of lipids in the rat. Gastroenterol. clin. biol., 3, $\mathrm{n}^{\circ} 2$.

BLOOM B., CHAIKOFF J. L., REINHARDT W. O., 1951. Intestinal lymph as pathway for transport of absorbed fatty acids of different chain lengths. Amer. J. Physiol., 166, 451-455.

BLOOMSTRAND R., 1955. Transport form of decanoic acid $1{ }^{14} \mathrm{C}$ in the lymph during intestinal absorption in the rat. Acta physiol. scand., 34, 67-70.

CARLIER H., BÉZARD J., 1975. Electron microscope autoradiographic study of intestinal absorption of decanoic and octanoic acids in the rat. J. Cell Biol., 65, 383-397.

DELSAL J. L., 1944. Nouveau procédé d'extraction des lipides du sérum par le méthylal. Applications aux microdosages du cholestérol total, des phosphoaminolipides ef des protéines. Bull. Soc. Chim. biol., 26, 99-105.

ELOY R., RAUL F., POUSSE A., MIRHOM R., ANANNA A., GRENIER J. F., 1977. Ex Vivo vascular perfusion of isolated rat small bowe. Eur. Surg. Res., 9, 96-112.

GLICKMAN R. M., KIRSCH K., ISSELBACHER K. J., 1972. Fat absorption during inhibition of protein synthesis : studies of lymph chylomicrons. J. clin. Invesf. 51, 356-363.

HYUN S. A., VAHOUNY G. V., TREADWELL C. R., 1967. Portal absorption of fatty acids in lymphand portal-vein cannulated rats. Biochim. biophys. Acta, 137, 296-305.

REDGRAVE T. G., 1969. Inhibition of protein synthesis and absorption of lipid into thoracic duct lymph of rats. Proc. Soc. exp. Biol. Med., 130, 776-780. 